\title{
CLIV.-2:3:5-Trinitro-4-aminophenol and Derivatives.
}

By Raphael Meldola, F.R.S., and James Gordon Hay, A.I.C.

THE acetyl derivative of the above compound has been fully described in a series of papers published during the last few years (Trans., 1906, $89,1935 ; 1907,91,1477 ; 1908,93,1659 ; 1909,95,1033)$, but the corresponding trinitroaminophenol has only been incidentally referred to (loc. cit., 91, 1477). As no trinitro-derivative of paraaminophenol has hitherto been prepared, and as the acetyl derivative has proved to be of especial interest on account of its reactivity, the parent trinitro-compound seemed worthy of detailed study from several points of view. In the first place, with respect to the possibility of catenation products being formed from this compound by interaction with amines in the same way as with the acetyl derivative, it may be pointed out that, as no ring-formation would be possible in this case, the products would be ortho-amino-derivatives of substituted diaryl- or arylalkyl-amines :

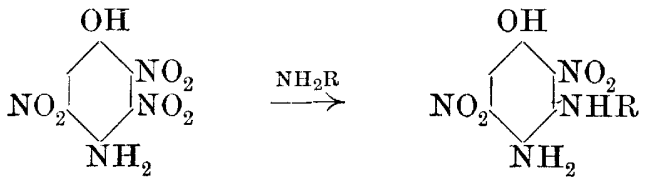

Compounds of this type have hitherto been but little studied on account of the difficulty of preparing them, and it was therefore hoped that the new trinitroaminophenol would prove to be a convenient material for synthesising them according to the above scheme. Our experiments in this direction have, however, so far led to negative results, and no definite compound has been obtained by the interaction of trinitroaminophenol and amines under any conditions. The addition of the amine to the alcoholic solution of the trinitro-compound leads in the first place to the formation of a highly coloured (generally violet) salt, which rapidly decomposes at the ordinary temperature. The trinitro-compound is apparently completely decomposed by amines, with the formation of resinous products. Although the study of these products of decomposition has for the present been abandoned, the research will be continued at some future time, as it is not improbable that by modifying the conditions under which the reaction takes place the required catenation products may be obtained.

The other direction in which the trinitroaminophenol has been studied is its behaviour on diazotisation, as the conditions are apparently most favourable for the elimination of a nitro-group in accordance with the rule which has been found to hold good in the case of the dinitroanisidines. It will be seen in the experimental part of this 
View Online paper that the 3-nitro-group in the trinitro-compound is not eliminated on diazotisation, a fact of considerable interest when considered in connexion with the results made known in a former paper (Trans., 1907, 91, 1482). It was shown in this last paper that $2: 3$-dinitro4-aminophenol does not lose the 3-nitro-group on diazotisation, although the latter is in a favourable ortho-position with respect to the amino-group. From this fact it was concluded that it is the methoxy-group which is the "loosening" substituent in this and analogous cases, since the corresponding dinitroanisidine readily parts with the 3 -nitro-group on diazotisation (loc. cit., p. 1484). This conclusion receives confirmation from the fact made known in the present paper that $2: 3: 5$-trinitro-4-aminophenol also retains the 3-nitro-group on diazotisation, since in this case the aminogroup (and therefore the diazonium group) is ortho with respect to two nitro-groups. The corresponding trinitro- $p$-anisidine has not been obtained by us, although attempts have been made to prepare this compound by the direct methylation of the silver salt of the trinitroacetylaminophenol (Trans., 1906, 89, 1937), and by the extreme nitration of aceto- $p$ anisidide. In the mean time a trinitroanisidine of the required constitution appears to have been obtained in small quantity by Reverdin by the extreme nitration of benzo-p-anisidide in acetic anhydride solution (Arch. Sci. phys. nat, 1909, 27, 395).

The process originally described for the preparation of trinitroacetyl-p-aminophenol (Trans., 1906, 89, 1936) has in the course of our work been considerably improved, and we are now enabled to give the details of the method finally adopted. In the earlier process the diacetyl derivative of $p$-aminophenol was nitrated so as to furnish as the first product the monoritro-compound of Hähle, and this by further nitration was converted into the trinitroacetylaminophenol. In a subsequent communication we pointed out that if the mononitrocompound is partially hydrolysed so as to convert it into the 3-nitro4-acetylaminophenol, and the latter nitrated under regulated conditions, 2 : 3-dinitro-4-acetylaminophenol is formed (Trans., 1907, 91, 1481). In this last paper it was also stated that by extreme nitration the mononitroacetylaminophenol is converted into the 2:3:5-trinitrocompound. The formation of the latter from 2:3-dinitro-4-acetylaminophenol as well as from $3: 5$-dinitro-4-acetylaminophenol (Trans., $1906,89,1936$ ) thus furnishes an additional proof of the constitution which we originally assigned to this compound :

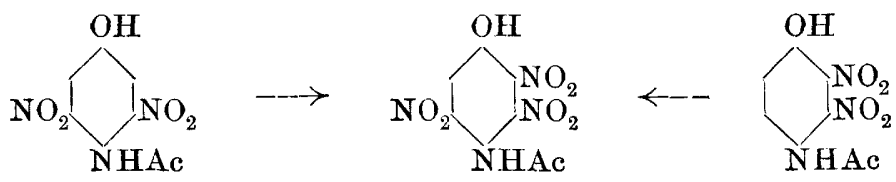


From these results it appears, also, that the course of View Online different, according as the mono- or the di-acetyl derivative of the mononitro-compound is used as the initial stage, although the final product is the same in both cases. The orienting influence of the $O$-acetyl group thus determines the entry of the second nitro-group into position 5, leaving only position 2 (or 6 ) for the third nitro-group. The hydroxyl group, on the other hand, sends the second nitro-group into position 2, leaving positions 5 and 6 for the third nitro-group, which in fact enters position 5 .

\section{Experimental.}

Preparation and Properties of Trinitroacetylaminophenol.

3-Nitro-4-acetylaminophenol is prepared by bydrolysing the diacetyl derivative in the way previously described (Trans., 1907, 91, 1481, footnote). The success of this operation depends on the rapidity with which the hydrolysis is effected, and the use of just sufficient alkali to bring about solution at the ordinary temperature. If an excess of alkali is present, or if the solution when once formed is not immediately acidified, there is much loss of material, owing to the decomposing action of the alkali. The 3-nitroacetylamino-compound, after precipitation, is collected, washed with water, dried, and further nitrated by dissolution in fuming nitric acid (D 1.5). One hundred c.c. of acid will nitrate 30 grams of the mononitro-compound, the latter being gradually stirred into the acid kept cool in melting ice. When all the substance has been dissolved in the acid, the solution is at once poured on to ice, and the trinitro-compound collected as soon as it has separated. As this last compound is decomposed on long standing in presence of dilute nitric acid, it is advisable to collect the precipitate and wash it free from acid with as little delay as possible. Owing to the solubility of the trinitro-compound in water, the quantity of ice used for precipitation must also be limited to a minimum, or considerable loss of material is incurred. The best solvent for purifying the crude product is acetic acid, one crystallisation being generally sufficient to give a pure compound if the above method of preparation is followed.

Some of the general properties of $2: 3: 5$-trinitro-4-acetylaminophenol have been described in former papers. The extreme reactivity of the compound renders it unsafe to dry it in contact with organic matter, such as filter paper. If dried in the water-oven on a paper filter, deflagration takes place, although it may be safely dried in contact with glass or porcelain. The extreme readiness with which the 3-nitrogroup is eliminated renders it impossible to prepare salts by combining the compound with strong bases, as the latter cause complete decom- 
position Attempts to replace the mobile nitro- View Online ethoxy-groups by interaction with sodium methoxide or ethoxide only resinous products. The extremely acid character of the compound enables it to decompose metallic salts containing weaker acid radicles, and this property has enabled us to isolate the potassium and sodium salts. The former was prepared by dissolving the trinitro-compound in a warm solution of potassium cyanide or potassium acetate; the salt separates out on cooling in splendid scarlet needles, which are very soluble in cold water, but which, by rapid washing, can be freed from mother-liquor. The salt was dried at the ordinary temperature in a desiccator, as it deflagrates when heated in the water-oven :

0.0316 gave $0.0082 \mathrm{~K}_{2} \mathrm{SO}_{4}$. $\mathrm{K}=11.64$.

$$
\mathrm{C}_{8} \mathrm{H}_{5} \mathrm{O}_{8} \mathrm{~N}_{4} \mathrm{~K}, \mathrm{H}_{2} \mathrm{O} \text { requires } \mathrm{K}=11 \cdot 43 \text { per cent. }
$$

The water of crystallisation could not be determined directly by drying for the reason above stated. The sodium salt was not obtained in sufficient quantity for analysis, as its solubility is greater than that of the potassium salt, and it could not be purified by washing. In appearance it resembles the potassium salt. This method of obtaining salts of the trinitro-compound appears to be generally applicable, and the research is being continued in this direction.

\section{Preparation and Properties of Trinitroaminophenol.}

The acetyl derivative cannot be hydrolysed by alkalis, owing to its instability in presence of such reagents. The early attempts to hydrolyse by means of sulphuric acid also failed, as the special. properties of the compound were not at the time sufficiently familiar (Trans., 1906, 89, 1937). We have now found that the acetyl group can be completely removed by sulphuric acid and without any decomposition of the trinitroamino-compound if the following method is closely adhered to.

The acetyl derivative is dissolved by stirring in an excess of concentrated sulphuric acid at the ordinary temperature of the laboratory. The vessel containing the solution is then plunged into a bath of boiling water for a few minutes and immediately withdrawn. The course of the hydrolysis must be followed by rapid tests, a drop of the solution being withdrawn on a glass rod at short intervals and diluted with water on a watch-glass. When hydrolysis is complete, a scarlet, crystalline precipitate is formed, and the sulphuric acid solution must be cooled at once by immersion in cold water, or the hydrolysis proceeds too far and decomposition takes place. A brownish tinge shown by the precipitate indicates that the action of the sulphuric acid has been too prolonged, as the pure compound is bright red. The whole operation takes but a few minutes, and its success depends upon arresting the 
action of the acid at the critical stage. When the latter View Online been reached and the solution cooled, the trinitroamino-compound is precipitated by pouring the solution on to ice. The red, crystalline deposit can be collected and washed with water until free from acid. It cannot be dried at $100^{\circ}$ without decomposition. It can be crystallised in small batches from acetic acid, from which it separates in deep red needles having a decomposing point of about $145^{\circ}$ :

0.0671 gave 12.65 c.c. $\mathrm{N}_{2}$ (moist) at $9 \cdot 5^{\circ}$ and $767 \mathrm{~mm} . \quad \mathrm{N}=22.84$. $\mathrm{O}_{6} \mathrm{H}_{4} \mathrm{O}_{7} \mathrm{~N}_{4}$ requires $\mathrm{N}=22.96$ per cent.

The compound is but feebly basic, the sulphate being completely dissociated by water. The salts formed with ammonia, alkyl- and aryl-amines, alkaline hydroxides, etc., are of an intense violet colour, but are so unstable that they undergo immediate decomposition.

\section{Diazotisation of the Trinitroaminophenol.}

The compound does not diazotise satisfactorily in acetic acid solution, the amino-group being no doubt "protected" by the nitrogroups on either side. In order to effect diazotisation, the substance is dissolved at the ordinary temperature in concentrated sulphuric acid, the solution diluted with a little water, and, when cold, an excess of solid sodium nitrite stirred in in small portions. The course of the diazotisation can be followed by mixing a drop of the solution with water on a watch-glass; the diazonium salt is decomposed by water with the precipitation of a bright yellow diazo-oxide (quinonediazide), so that the appearance of a yellow instead of a red precipitate on dilution indicates the completion of the reaction.

\section{$2: 3: 5$-Trinitrophenol.}

The solution of the diazonium sulphate prepared as above undergoes the usual "diazo-decompositions" with various reagents. On boiling with excess of absolute alcohol as long as nitrogen is evolved, evaporating the solvent, and diluting with water, trinitrophenol crystallises out in ochreous needles. This compound, which is of interest as being an isomeride of picric acid, has been briefly referred to in a recent note $(J$. Soc. Dyers, 1909, 25, 12). It is slightly soluble in boiling water, from which it separates in yellow needles, melting at $119-120^{\circ}$, and becoming ochreous and opaque on drying at $100^{\circ}$. It is readily soluble in alcohol, acetic acid, or benzene :

0.0852 gave 13.35 c.c. $\mathrm{N}_{2}$ (moist) at $17^{\circ}$ and $764 \mathrm{~mm} . \quad \mathrm{N}=18.24$. $\mathrm{C}_{6} \mathrm{H}_{3} \mathrm{O}_{7} \mathrm{~N}_{3}$ requires $\mathrm{N}=18.34$ per cent.

This trinitrophenol dyes silk and wool with a more orange shade than picric acid. The hydroxyl group is so effectively protected that 

the compound could not be satisfactorily methylated or acetylated by any of the ordinary methods. On dissolving in hot potassium carbonate solution, a potassium salt separates out on cooling in red needles, which contain no water of crystallisation :

$0 \cdot 1672$ gave $0 \cdot 0554 \mathrm{~K}_{2} \mathrm{SO}_{4} . \quad \mathrm{K}=14 \cdot 81$.

$\mathrm{C}_{6} \mathrm{H}_{2} \mathrm{O}_{7} \mathrm{~N}_{3} \mathrm{~K}$ requires $\mathrm{K}=14 \cdot 66$ per cent.

Other salts were prepared and examined qualitatively. When dry, they deflagrate on heating, and are not at all comparable in explosive power with the picrates. The solutions of the trinitrophenol are much more bighly coloured than the solid compound, a property which, taken in connexion with the deep red colour of the salts, suggests the usual isonitro-tautomerism :

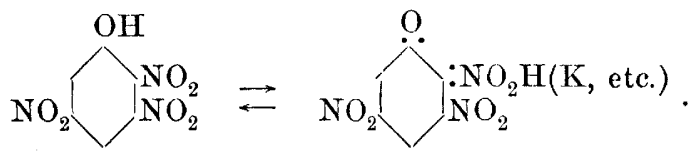

The trinitrophenol appears to form a molecular compound with naphthalene, which crystallises from alcohol in orange needles melting at $100-101^{\circ}$. This compound is, however, much less stable than the corresponding picrate, and the successive analyses following the crystallisations indicated that dissociation takes place in alcoholic solution.

From the foregoing results, it follows that there is no loss of a nitro-group on diazotisation.

\section{$2: 3: 5$-Trinitroquinonediazide,}
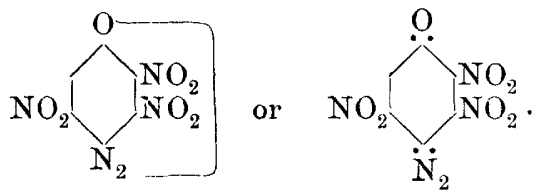

The diazonium sulphate prepared as described is immediately decomposed on dilution with water, with the formation of a quinonediazide, which separates as a dense, yellow, micro-crystalline powder. After being washed free from acid, the compound, although highly explosive, can be safely dried in the water-oven. It has been found very difficult to obtain the substance perfectly pure, as it is decomposed by most solvents, and tends to retain those which do not interact with it chemically. The analytical results are, however, perfectly definite in establishing the fact that no loss of a nitro-group occurs on the transformation of the diazonium salt into the quinonediazide. The latter is, in fact, unchanged by a solution of sodium acetate at the ordinary temperature, although complete decomposition with the 
formation of resinous products is caused by the action of View. Online alkalis or of alkaline carbonates. The compound can be crystallised from boiling glacial acetic acid, provided the quantity is limited to about half a gram. Boiling with this solvent causes some amount of decomposition, as shown by the evolution of nitrous fumes, but the loss is not serious if the scale of working is not too large. From the hot saturated solution, the compound separates on cooling in dense, ochreous prisms, the decomposing point of which was not determined on account of the highly explosive character of the substance. Analysis indicates that all the nitro-groups are retained :

$$
\begin{gathered}
0.0574 \text { gave } 13.05 \text { c.c. } \mathrm{N}_{2} \text { (moist) at } 13^{\circ} \text { and } 765 \mathrm{~mm} . \mathrm{N}=26.98 \text {. } \\
0.0574 \Rightarrow 13 \cdot 23, \mathrm{~N}_{2}, " 14^{\circ}, 764.3 \mathrm{~mm} . \quad \mathrm{N}=27 \cdot 25 . \\
\mathrm{C}_{6} \mathrm{HO}_{7} \mathrm{~N}_{5} \text { requires } \mathrm{N}=27 \cdot 45 \text { per cent. }
\end{gathered}
$$

This quinonediazide couples with $\beta$-naphthol with extreme difficulty, a property which is probably due to the protective action of the 3- and 5-nitro-groups on the intermediate diazo-group. In the presence of pyridine or an alkali, an azo-compound is formed, but there is also further decomposition, due to the elimination of one of the nitro-groups, the mobility of which is increased by the proximity of the azo-naphthol complex. The azo-compound thus formed appears to be a mixture of extremely insoluble products, which will require further study before any definite conclusion concerning their nature can be drawn.

The 3-nitro-group in the quinonediazide being in a favourable position for displacement, attempts were made to arrive at definite products by the substitution of other radicles for this group. Boiling with sodium acetate solution or with absolute alcohol brings about definite decomposition, the latter in a quite unexpected way.

\section{Dinitrohydroxyquinonediazide,}
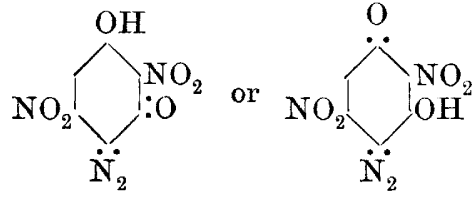

(or the corresponding cross-linking).

The trinitro-compound dissolves in a strong boiling solution of sodium acetate without any considerable evolution of nitrogen, and, on cooling, a dense, orange, crystalline sodium salt separates. The latter was collected, washed, redissolved in a small quantity of hot water, and acidified with hydrochloric acid. Large, golden scales separated on cooling, and these had all the properties of a quinonediazide, being highly explosive and coupling readily with $\beta$-naphthol 
in presence of alkali. From this result it appears that the 3-nitrogroup is more readily removed than the diazo-group :

0.0614 gave 13.05 c.c. $\mathrm{N}_{2}$ (moist) at $18^{\circ}$ and $767 \cdot 1 \mathrm{~mm}$. $N=24.76$. $\mathrm{C}_{6} \mathrm{H}_{2} \mathrm{O}_{6} \mathrm{~N}_{4}$ requires $\mathrm{N}=24 \cdot 78$ per cent.

The compound may have either of the above formulæ. Although the ortho-quinonediazide must be the first product resulting from the displacement of the 3-nitro-group, it may easily undergo isomeric transformation into the para-compound if the latter configuration is the more stable. In view of the explosive character of the compound, we are inclined to believe that it possesses the para-constitution, as the ortho-quinonediazides are not, as a rule, so highly explosive. It is, however, impossible at present to decide conclusively between these formulx.

The quinonediazide in a pasty and moist condition combines with $\beta$-naphthol dissolved in sodium hydroxide, giving a highly-coloured and dense, crystalline deposit of a sodium salt having a bronzy lustre. This salt can be collected and washed with a dilute solution of sodium hydroxide until free from excess of naphthol. The azo-compound, liberated by hydrochloric acid from the sodium salt, dissolves very sparingly in boiling glacial acetic acid, from which it separates on cooling in dull red needles, which begin to blacken about $240^{\circ}$, but do not melt at $320^{\circ}$. The azo-compound is phenolic in character, dissolving in aqueous ammonia or very dilute sodium hydroxide with an indigo-blue colour, which changes to red on adding more sodium hydroxide. This colour change most probably indicates the formation of a disodium salt from a mono-sodium salt, as there are two hydroxyl groups in the molecule, and it seems unnecessary in such a case to introduce any hypothesis of structural change on the chemical evidence only. From its mode of formation. the compound must be an azonaphthol derivative of $2: 5$-dinitror esorcinol :

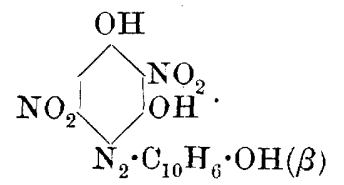

0.070 gave 8.95 c.c. $\mathrm{N}_{2}$ (moist) at $13^{\circ}$ and $759 \cdot 7 \mathrm{~mm}$. $\quad \mathrm{N}=15 \cdot 1$.

$\mathrm{C}_{16} \mathrm{H}_{10} \mathrm{O}_{7} \mathrm{~N}_{4}$ requires $\mathrm{N}=15 \cdot 14$ per cent.

Concentrated sulphuric acid dissolves the compound with a reddishviolet colour, which becomes redder, and finally gives an orange, gelatinous precipitate on dilution with water. It is probable that this same azo-compound is among the products of the action of the trinitroquinonediazide on $\beta$-naphthol in presence of alkali.

The decomposition of the trinitroquinonediazide by boiling absolute alcohol leads to the formation of a compound the analysis of which

voL. XCV.

$4 \mathrm{x}$ 
indicates that a nitro-group is replaced by the ethoxy-group. Online study of this reaction is being continued.

The cost of this research has been partly met by a grant from the Government Grant Fund of the Royal Society, a source of assistance which we have much pleasure in acknowledging.

Finsburt Technical College. 